\title{
ON THE FUNDAMENTAL GROUP OF THE COMPLEMENT OF ARRANGEMENTS
}

\author{
By Nguyen Viet Dung
}

Let $V$ be a vector space of finite dimension. An arrangement of hyperplanes in $V$ is a finite collection $\mathcal{A}$ of hyperplanes of $V$. An arrangement $\mathcal{A}$ will be said to be real (resp. complex) if $V$ is a real (resp. complex) vector space. The complexification of a hyperplanes $H$ of $\mathbf{R}^{n}$ is the hyperplane $H_{\mathbf{C}}$ of $\mathbf{C}^{n}$ having the same equation as $H$. Given an arrangement $\mathcal{A}$ in $\mathbf{R}^{n}$, we have its complexification $\mathcal{A}_{\mathbf{C}}$ to be the complex arrangement $\left\{H_{\mathbf{C}} ; H \in \mathcal{A}\right\}$ in $\mathbf{C}^{n}$.

Given an arrangement $\mathcal{A}$, we are interested in finding a presentation for the fundamental group $\pi_{1}(M)$ of the complement

$$
M=V-\bigcup_{H \in \mathcal{A}} H
$$

in case $\mathcal{A}$ is a complex arrangement, and $\pi_{1}\left(M_{\mathbf{C}}\right)$ of the complement of $\mathcal{A}_{\mathbf{C}}$ in case $\mathcal{A}$ is a real arrangement. In [2] we have suggested a geometrical method to compute the fundamental group of a manifold equipped with a suitable cellular decomposition. Also, given a real arrangement $\mathcal{A}$ in $\mathbf{R}^{n}$, we have introduced a certain cellular decomposition $\mathcal{C}\left(\mathbf{C}^{n}, \mathcal{A}\right)$ of $\mathbf{C}^{n}$, induced from the arrangement $\mathcal{A}$. In this note, we will apply our method to this decomposition to find a presentation for $\pi_{1}\left(M_{\mathbf{C}}\right)$ of any real arrangement $\mathcal{A}$. Such a presentation has been given by $M$. Salvetti in [4] using his complex. After reducing the problem to the case of dimension 2, W. Arvola has suggested an algorithm to find a presentation for the complement of a complex arrangement. In a sequent paper [3] we will also treat the case when $\mathcal{A}$ is a complex arrangement.

We first recall of our method suggested in [2]. Let $\mathcal{M}$ be a connected topological-manifold of dimension $\mathrm{n}$ with a locally finite $\mathrm{CW}$-semicomplex structure $\mathcal{C}_{\mathcal{M}}$ such that $\mathcal{M}$ is 1-codimensionally regular (see [2] for the notion of $\mathrm{CW}$-semicomplex and 1codimensional regularity). Each $(n-1)$-cell $\sigma$ of $\mathcal{M}$ is a face of exactly two $n$-cells, say $c$ and $c^{\prime}$. Then we have two $n$-intervals $\left[c, \sigma, c^{\prime}\right]$ and $\left[c^{\prime}, \sigma, c\right]$. We specify one of them by $[\sigma]$ and the other by $[\sigma]^{-1}$. A $n$-path $\gamma$ on $\mathcal{M}$ is a join of a finite number of $n$-intervals

$$
\gamma=\left[\sigma_{1}\right]^{\epsilon_{1}} \vee\left[\sigma_{2}\right]^{\epsilon_{2}} \vee \cdots \vee\left[\sigma_{k}\right]^{\epsilon_{k}}
$$

where $\epsilon_{\imath}= \pm 1, \sigma_{\imath}$ are $(n-1)$-cells of $\mathcal{M}, 1 \leq i \leq k$. If $\left[\sigma_{1}\right]^{\epsilon_{1}}=\left[c, \sigma_{1}, c_{1}\right]$ and $\left[\sigma_{k}\right]^{\epsilon_{k}}=$ $\left[c_{k}, \sigma_{k}, c^{\prime}\right]$, for some $n$-cells $c, c_{1}, c_{k}$ and $c^{\prime}$ we say that $\gamma$ is a $n$-path from $c$ to $c^{\prime}$. Among $n$-paths on the manifold $\mathcal{M}$ we have defined in [2] a certain equivalence relation.

Let $\mathcal{M}$ be given a base point $*$ belonging to a certain $n$-cell $c_{0}$. Then, the equivalence classes of closed $n$-paths at $*$ form a group denoted by $\pi_{1}\left(\mathcal{C}_{\mathcal{M}}, *\right)$. In [2] we have proved the isomorphism $\pi_{1}\left(\mathcal{C}_{\mathcal{M}}, *\right) \cong \pi_{1}(\mathcal{M}, *)$. So, in order to compute $\pi_{1}(\mathcal{M}, *)$, it suffices to compute $\pi_{1}\left(\mathcal{C}_{\mathcal{M}}, *\right)$. And the latter can be determined by means of the decomposition $\mathcal{C}_{\mathcal{M}}$ as below

Received June 28, 1993. 
We call a $n$-tree $T$ of $\mathcal{M}$ a family of $n$-cells and $(n-1)$-cells of $\mathcal{C}_{\mathcal{M}}$ such that each $(n-1)$-cell of $T$ is a face of exactly two $n$-cells of $T$ and the union of all cells in $T$ is simply connected. A $n$-tree $T$ is maximal if it is not contained in any othe $n$-tree. It is easily seen that such a maximal $n$-tree exists and it contains all $n$-cells of $\mathcal{C}_{\mathcal{M}}$. Let $K$ be a subspace of $\mathcal{M}$ which is the underlying space of a $\mathrm{CW}$-subsemicomplex of codimension 2 of $\mathcal{M}$. Then we have

THEOREM 1 (see [2]). Let $\mathcal{M}$ be a connected manifold with a 1-codimensionally regular $C W$-semicomplex structure $\mathcal{C}_{\mathcal{M}}$ and $T$ a maximal $n$-tree of $\mathcal{C}_{\mathcal{M}}$. Let $K$ as above. Then $\pi_{1}(\mathcal{M}-K, *)$ has a presentation with generators $g_{s}$ indexed by the set $\mathcal{M}^{(n-1)}$ $\mathcal{M}^{(n-2)}$ of all $(n-1)$-cells of $\mathcal{M}$, with following defining relations

(i) $g_{\sigma}=1 \quad$ if $\sigma \in T$

(ii) $g_{\sigma_{1}}^{\epsilon_{1}} \cdot g_{\sigma_{2}}^{\epsilon_{2}} \cdots g_{\sigma_{q}}^{\epsilon_{q}}=1$ if $\left[\sigma_{1}\right]^{\epsilon_{1}} \vee\left[\sigma_{2}\right]^{\epsilon_{2}} \vee \cdots \vee\left[\sigma_{q}\right]^{\epsilon_{q}}$ is well defined and is a closed $n$-path around some $(n-2)$-cell of $\mathcal{M}-K$, i.e. $\sigma_{1}, \ldots, \sigma_{q}$ having a common $(n-2)$-face in $\mathcal{M}-K$.

Next we recall of the decomposition $\mathcal{C}\left(\mathbf{C}^{n}, \mathcal{A}\right)$ induced from the arrangement $\mathcal{A}=$ $\left\{H_{s} ; s \in I\right\}$ of hyperplanes in a $n$-dimensional real vector space $V=\mathbf{R}^{n}$. For each $s \in I$ we have a linear function $u_{s}: V \rightarrow \mathbf{R}$ with $H_{s}=\operatorname{ker} u_{s}$. Clearly, $V-H_{s}$ consists of two components $V_{s}^{+}=\left\{x \in V: u_{s}(x)>0\right\}$ and $V_{s}^{-}=\left\{x \in V ; u_{s}(x)<0\right\}$. In a natural way, the arrangement $\mathcal{A}$ induces a cellular decomposition $\mathcal{C}(V, \mathcal{A})$ of $V$. An arbitrary cell of $\mathcal{C}(V, \mathcal{A})$ is an equivalence class with respect to the following equivalence relation

" $x$ and $y$ are equivalent if $u_{s}(x) \cdot u_{s}(y)>0$ or $u_{s}(x)=u_{s}(y)=0$ for all $s \in I$."

It is easily seen that a $n$-cell of $\mathcal{C}(V, \mathcal{A})$ is a connected component of $V-\cup_{s \in I} H_{s}$ and is usually called a chamber of $\mathcal{A}$. A cell of dimension lower than $\mathrm{n}$ is contained in the closure $\bar{c}$ of a certain chamber $c$. The closure of such a cell is the intersection $\bar{c} \cap\left\{\bigcap_{j=1}^{l} H_{s_{z}}\right\}$ of $\bar{c}$ and some hyperplanes of $\mathcal{A}$. We denote this cell by $c_{s_{1}, \ldots, s_{l}}$. For each cell $e$ of $\mathcal{C}(V, \mathcal{A})$ we denote by $|e|$ the intersection of all hyperplanes of $\mathcal{A}$ containing $e$ and call it the support of $e$. Then it is easily seen that $\left|c_{s_{1}, \ldots, s_{l}}\right|=\bigcap_{j=1}^{l} H_{s_{j}}$.

Let us consider the complexification $\mathbf{C}^{n}=V+\iota V$ of $V$, where $\iota$ denotes $\sqrt{-1}$, the imaginary unit. Then we have the complexification of the arrangement $\mathcal{A}, \mathcal{A}_{\mathbf{C}}=$ $\left\{H_{s}+\iota H_{s} ; s \in \cdot I\right\}$, in $\mathbf{C}^{n}$ with the complement $M_{\mathbf{C}}=V_{\mathbf{C}}-\cup_{s \in I}\left(H_{s}+\iota H_{s}\right)$. The arrangement $\mathcal{A}$ induces also a cellular decomposition $\mathcal{C}\left(\mathbf{C}^{n}, \mathcal{A}\right)$ of $\mathbf{C}^{n}$ as follows.

(a) An arbitrary $2 n$-cell in $\mathcal{C}\left(\mathbf{C}^{n}, \mathcal{A}\right)$ is of the form $c+\iota V$, where $c$ is a chamber of $\mathcal{C}(V, \mathcal{A})$

(b) An arbitrary $(2 n-1)$-cell in $\mathcal{C}\left(\mathbf{C}^{n}, \mathcal{A}\right)$ is of the form $c_{s}+\iota V_{s}^{\epsilon}, \epsilon=+$ or - , with $c_{s}$ and $V_{s}^{\epsilon}$ as in the above notations.

(c) A cell of dimension $\leq 2 n-1$ is of the form

$$
c_{s_{1}, \ldots, s_{k}}+\iota D
$$

where $D$ is any cell in $\mathcal{C}\left(V,\left\{H_{s_{1}}, \ldots, H_{s_{k}}\right\}\right)$.

Remark 2. Denoting $V_{s_{1}, \ldots, s_{k}}^{\epsilon_{1}, \ldots, \epsilon_{k}}$ to be the intersection $V_{s_{1}}^{\epsilon_{1}} \cap \ldots \cap V_{s_{k}}^{\epsilon_{k}}$, with $s_{\jmath} \in I, \epsilon_{\jmath}=$ + or - , we observe that any $(2 n-2)$-cell of $\mathcal{C}\left(\mathbf{C}^{n}, \mathcal{A}\right)$ lying in the complement $M$ will be of the form

$$
c_{s_{1}, \ldots, s_{k}}+\iota V_{s_{1}, \ldots, s_{k}}^{\epsilon_{1}, \ldots, \epsilon_{k}}
$$


where $c_{s_{1}, \ldots, s_{k}}$ is a cell of codimension 2 of $\mathcal{C}(V, \mathcal{A})$.

Now we will use theorem 1 and this decomposition $\mathcal{C}\left(\mathbf{C}^{n}, \mathcal{A}\right)$ to give a presentation for the group $\pi_{1}\left(M_{\mathbf{C}}, *\right)$. According to Theorem 1, we have a presentation of the fundamental group of the complement $\pi_{1}(M)$ with generators corresponding to $(2 n-1)$-cells of $\mathcal{C}\left(\mathbf{C}^{n}, \mathcal{A}\right)$ and with defining relations given by $(2 n-2)$-cells of $\mathcal{C}\left(\mathbf{C}^{n}, \mathcal{A}\right)$ lying in $M$ modulo a chosen maximal $2 n$-tree $T$. We will denote by $g_{\sigma}^{+}$and $g_{\sigma}^{-}$the generators corresponding to $\sigma+\iota V_{s}^{+}$and $\sigma+V_{s}^{-}$respectively, where $\sigma$ is any $(n-1)$-cell of $\mathcal{C}(V, \mathcal{A})$ with the support $|\sigma|=H_{s} \in \mathcal{A}$. Now we try to simplify this presentation.

LEMma 3. We can choose a maximal $2 n$-tree $T_{0}$ of $\mathcal{C}\left(\mathbf{C}^{n}, \mathcal{A}\right)$ so that in the group $\pi_{1}(M)$ we have $g_{\sigma}^{-}=1$ for any $(n-1)$-cell $\sigma$ of $\mathcal{C}(V, \mathcal{A})$.

Let $T$ be the maximal $2 n$-tree as in Lemma 3 . According to Theorem 1 (i), the fundamental group $\pi_{1}(M)$ has the set of generators $\left\{g_{\sigma}^{+} ; \sigma\right.$ is a $(n-1)$-cell of $\left.\mathcal{C}(V, \mathcal{A})\right\}$. For the sake of simplicity, from now on, we will denote $g_{\sigma}^{+}$simply by $g_{\sigma}$. We can also prove that two generators corresponding to $(n-1)$-cells of $\mathcal{C}(V, \mathcal{A})$ having the same support are conjugate. So, there are as many generators as there are hyperplanes in $\mathcal{A}$. Denote the generator corresponding to the hyperplane $H_{s}$ by $g_{s}, s \in I$.

Next we consider the defining relations of the group $\pi_{1}(M)$. According to Theorem 1 (ii), these relations are given by $(2 n-2)$-cells in $M$. By Remark 2, any $(2 n-2)$-cell in $M$ will be of the form

$$
c_{s_{1}, \ldots, s_{k}}^{\epsilon_{1}, \ldots, \epsilon_{k}}=c_{s_{1}, \ldots, s_{k}}+\iota V_{s_{1}, \ldots, s_{k}}^{\epsilon_{1}, \ldots, \epsilon_{k}}
$$

where $\epsilon_{i}=+$ or,$- s_{i} \in I$ and $c_{s_{1}, \ldots, s_{k}}$ is a codimension 2 cell of $\mathcal{C}(V, \mathcal{A})$. We first observe that the $(2 n-1)$-cells of $\mathcal{C}\left(\mathbf{C}^{n}, \mathcal{A}\right)$ having $c_{s_{1}, \ldots, s_{k}}^{\epsilon_{1}, \ldots, \epsilon_{k}}$ as a common face are $\sigma_{1}+$ $\iota V_{s_{1}}^{\epsilon_{1}}, \ldots, \sigma_{k}+\iota V_{s_{k}}^{\epsilon_{k}}$, and $\sigma_{1}^{\prime}+V_{s_{1}}^{\epsilon_{1}}, \ldots, \sigma_{k}^{\prime}+\iota V_{s_{k}}^{\epsilon_{k}}$, where $\sigma_{\imath}, \sigma_{\imath}^{\prime}, 1 \leq i \leq k$, are codimension 1 cells of $\mathcal{C}(V, \mathcal{A})$ having $c_{s_{1}, \ldots, s_{k}}$ as a common face and $\left|\sigma_{i}\right|=\left|\overline{\sigma_{i}^{\prime}}\right|=H_{s_{i}}, 1 \leq i \leq k$. Looking at a 2-plane perpendicular to $c_{s_{1}, \ldots, s_{k}}^{\epsilon_{1}, \ldots, \epsilon_{k}}$ at an inner point of this cell, we obtain

LEMMA 4. The relation given by $c_{s_{1}, \ldots, s_{k}}^{\epsilon_{1}, \ldots, \epsilon_{k}}$ is

$$
g_{\sigma_{1}}^{\epsilon_{1}} \cdots g_{\sigma_{k}}^{\epsilon_{k}} \cdot\left(g_{\sigma_{1}^{\prime}}^{\epsilon_{1}}\right)^{-1} \cdots\left(g_{\sigma_{k}^{\prime}}^{\epsilon_{k}}\right)^{-1}=1
$$

Let us consider the codimension 2 cell $c_{s_{1}, \ldots, s_{l}}$ of $\mathcal{C}(V, \mathcal{A})$. Changing the sign of linear functions $u_{s_{i}}, 1 \leq i \leq l$, if necessary, we can assume that $V_{s_{1}, \ldots, s_{l}}^{+, \ldots,+}=V_{s_{1}}^{+} \cap \ldots \cap V_{s_{l}}^{+} \neq \emptyset$. Then in $\mathcal{C}\left(V_{\mathbf{C}}, \overline{\mathcal{A}}\right)$ we will have the following $(2 n-2)$-cells : $c_{s_{1}, \ldots, s_{l}}^{\epsilon_{1}, \ldots, \epsilon_{l}}$, where $\left(\epsilon_{1}, \ldots, \epsilon_{l}\right)$ runs over the set

$$
\{(+,+, \ldots,+,+),(-,+, \ldots,+,+), \ldots,(-,-, \ldots,-,+),(-,-, \ldots,-,-)\} .
$$

By a direct computation, from the defining relations of the group $\pi_{1}\left(M_{\mathbf{C}}\right)$ corresponding to these $(2 n-2)$-cells as determined in Lemma 4 we obtain the set of relations

$$
\left\{g_{\sigma_{1}} g_{\sigma_{2}} \cdots g_{\sigma_{l}}=g_{\sigma_{s(1)}} g_{\sigma_{s(2)}} \cdots g_{\sigma_{s(l)}} ; s \in C\right\}
$$

where $C$ is the set of cyclic permutations of $\{1, \ldots, l\}$. Denote this set of relations by $R\left(c_{s_{1}, \ldots, s_{l}}\right)$.

Next we consider two cells of codimension 2 of $\mathcal{C}(V, \mathcal{A})$ having the same support. Suppose they are $c_{s_{1}, \ldots, s_{l}}$ and $\tilde{c}_{s_{1}, \ldots, s_{l}}$. Then we have $(n-1)$-cells $\sigma_{1}, \ldots, \sigma_{l}$ and $\tilde{\sigma}_{1}, \ldots, \tilde{\sigma}_{l}$ 
around $c_{s_{1}, \ldots, s_{l}}$ and $\tilde{c}_{s_{1}, \ldots, s_{l}}$ respectively. Moreover $\left|\sigma_{i}\right|=\left|\tilde{\sigma}_{i}\right|=H_{s_{\imath}}, 1 \leq i \leq l$. We can prove that there is a class of $n$-paths $\lambda$ such that $\lambda g_{\tilde{\sigma}_{i}} \lambda^{-1} \simeq g_{\sigma_{i}}, 1 \leq i \leq l$. Combining this and relations $R\left(c_{s_{1}, \ldots, s_{l}}\right)$ and $R\left(\tilde{c}_{s_{1}, \ldots, s_{l}}\right)$ we see that $c_{s_{1}, \ldots, s_{l}}$ and $\tilde{c}_{s_{1}, \ldots, s_{l}}$ determine the same relation of the group $\pi_{1}(M)$.

Now substituting to $g_{\sigma_{i}}$ the right conjugate of $g_{s_{i}}$, the relations $R\left(c_{s_{1}, \ldots, s_{l}}\right)$ translate into the relations amongs $g_{s_{i}}$. We denote these relations by $R\left(s_{1}, \ldots, s_{l}\right)$.

So from above investigations we obtain

THEOREM 5. The fundamental group $\pi_{1}\left(M_{\mathbf{C}}\right)$ accepts a presentation with the set of generators $\left\{g_{s} ; s \in I\right\}$ and with definıng relation $R\left(s_{1}, \ldots, s_{l}\right)$ whenever $H_{s_{1}} \cap \ldots \cap H_{s_{l}}$ is of codimension 2 .

\title{
REFERENCE
}

[1] W. Arvola, The fundamental group of the complement of an arrangement of complex hyperplanes, to appear in Topology.

[2] N.V. Dung, The fundamental groups of the space of regular orbits of the affine Weyl groups, Topology 22 (1983), 425-435.

[ 3 ] N.V. Dung-H.H. Vui, Braid monodromy of arrangements of complex lines, (in preparation).

[4] M. Salvetti, Topology of the complement of real hyperplanes in $\mathbf{C}^{N}$, Inv. Math. 88 (1987), 603-618.

\author{
Hanol Institute of Mathematics \\ P.O. Box 631 Bo Hô \\ 10000 HANOI \\ VIETNAM
}

